\title{
Development and characterization of cholangioids from normal and diseased human cholangiocytes as an in vitro model to study primary sclerosing cholangitis
}

\author{
Lorena Loarca1,2, Thiago M De Assuncao 1,2, Nidhi Jalan-Sakrikar', Steve Bronk1, Anuradha Krishnan', Bing Huang1, \\ Leslie Morton ${ }^{1}$, Christy Trussoni ${ }^{1}$, Lorena Marcano Bonilla', Eugene Krueger ${ }^{1}$, Steve O'Hara ${ }^{1}$, Patrick Splinter ${ }^{1}$, \\ Guang Shi', María José Lorenzo Pisarello', Gregory J Gores ${ }^{1}$, Robert C Huebert ${ }^{1,3}$ and Nicholas F LaRusso ${ }^{1,3}$
}

Primary sclerosing cholangitis (PSC) is an incurable, fibroinflammatory biliary disease for which there is no effective pharmacotherapy. We recently reported cholangiocyte senescence as an important phenotype in PSC while others showed that portal macrophages accumulate in PSC. Unfortunately, our ability to explore cholangiocyte senescence and macrophage accumulation has been hampered by limited in vitro models. Thus, our aim was to develop and characterize a three-dimensional (3D) model of normal and diseased bile ducts (cholangioids) starting with normal human cholangiocytes (NHC), senescent NHC (NHC-sen), and cholangiocytes from PSC patients. In 3D culture, NHCs formed spheroids of $\sim 5000$ cells with a central lumen of $\sim 150 \mu \mathrm{m}$. By confocal microscopy and western blot, cholangioids retained expression of cholangiocyte proteins (cytokeratin 7/19) and markers of epithelial polarity (secretin receptor and GM130). Cholangioids are functionally active, and upon secretin stimulation, luminal size increased by $\sim 80 \%$. Cholangioids exposed to hydrogen peroxide exhibited cellular senescence and the senescence-associated secretory phenotype (SASP; increased IL-6, p21, SA- $\beta-G a l, y H 2 A . x$ and $p 16$ expression). Furthermore, cholangioids derived from NHC-sen or PSC patients were smaller and had slower growth than the controls. When co-cultured with THP-1 macrophages, the number of macrophages associated with NHC-sen or PSC cholangioids was five- to seven-fold greater compared to co-culture with non-senescent NHC. We observed that NHC-sen and PSC cholangioids release greater number of extracellular vesicles (EVs) compared to controls. Moreover, conditioned media from NHC-sen cholangioids resulted in an $\sim 2$-fold increase in macrophage migration. In summary, we developed a method to generate normal and diseased cholangioids,

characterized them morphologically and functionally, showed that they can be induced to senescence and SASP, and demonstrated both EV release and macrophage attraction. This novel model mimics several features of PSC, and thus will be useful for studying the pathogenesis of PSC and potentially identifying new therapeutic targets.

Laboratory Investigation (2017) 97, 1385-1396; doi:10.1038/labinvest.2017.63; published online 11 September 2017

Cholangiocytes, the epithelial cells lining the biliary tree, not only have important physiologic functions, but also are the target of a group of diseases known as the cholangiopathies, one of which is primary sclerosing cholangitis (PSC), an incurable, idiopathic, and fibroinflammatory syndrome. ${ }^{1}$ Although the pathogenesis of PSC is unclear, we recently reported that cholangiocyte senescence is an important phenotype in patients with PSC. ${ }^{2}$ Moreover, Cameron et al ${ }^{3}$ reported the accumulation of perisinusoidal macrophages into the bile ducts of patients with PSC. Unfortunately, well defined in vitro models to pursue these observations and further interrogate the pathogenesis of PSC are limited. For example, studying biliary diseases with existing two-dimensional (2D), in vitro techniques on tissue culture dishes has shortcomings, including: (i) plastic surfaces are several orders of magnitude stiffer than the physiologic environment; ${ }^{4}$ (ii) tissue culturing on plastic surfaces does not recapitulate physiologic cell-cell and cell-microenvironment interactions; and (iii) on 2D plastic, it is not possible to observe a ductal lumen as well as changes in bile duct anatomy or physiology such as bile duct

\footnotetext{
${ }^{1}$ Division of Gastroenterology and Hepatology, Department of Medicine, Mayo Clinic, Rochester, MN, USA

Correspondence: Dr NF LaRusso, MD or Dr RC Huebert, MD, Division of Gastroenterology and Hepatology, Mayo Clinic, 200 First Street SW, Rochester, MN 55905, USA.

E-mail: larusso.nicholas@mayo.edu or huebert.robert@mayo.edu

${ }^{2}$ These authors contributed equally to this work.

${ }^{3}$ These authors contributed equally to this work.

Received 5 January 2017; revised 18 May 2017; accepted 23 May 2017
} 
obliteration. Recently, advances in the field of in vitro research have been made with the introduction of three-dimensional (3D) cell culturing techniques, as 3D cultures can be made to resemble the morphology of organs and recapitulate better in vivo biologic processes. ${ }^{5}$ Three-dimensional in vitro cellular clusters that exhibit spontaneous formation, growth, and polarity, termed organoids, have been generated from embryonic stem cells, pluripotent stem cells, as well as from a number of primary cell types. ${ }^{6-9}$ To date, several manuscripts have described the development of 3D cultures from cholangiocytes, the majority of them reporting the formation of 3D cultures of cholangiocytes that were differentiated from pluripotent or embryonic stem cells ${ }^{10-13}$ using this approach to model biliary diseases such as Alagille syndrome, polycystic liver disease, and cystic fibrosis. ${ }^{10,11}$ More recently, Spirli and colleagues generated organoids from cholangiocytes from mice with polycystic liver disease and studied the effects of adenylyl cyclase inhibition on organoid size. ${ }^{14}$ Also, Waisbourd-Zinman et $\mathrm{al}^{15}$ demonstrated a link between a plant-derived toxin and development of biliary atresia-associated cellular changes using 3D cultures of mature and freshly isolated extrahepatic neonatal mouse cholangiocytes. Previously, using collagen matrices, our group reported the formation of 3D hepatic cysts from bile ducts from normal rats and rats with polycystic kidney disease. ${ }^{16}$ Thus, to extend and refine these efforts, our objectives here were to: (i) develop a simple, reproducible 3D in vitro model of normal human and diseased cholangiocytes, which we have termed cholangioids, beginning with cholangiocyte cell lines; (ii) characterize the morphology, size, cell number, and polarity of the cholangioids; (iii) assess the functionality of the cholangioids; and (iv) begin to utilize our model to mimic selected features of PSC, such as cellular senescence and macrophage recruitment. Herein, we describe a novel, in vitro 3D model of PSC, which will be useful to understand the mechanisms of PSC pathogenesis, the interactions between PSC bile ducts and immune cells, and potentially to develop new therapeutic targets.

\section{MATERIALS AND METHODS}

The normal human cholangiocyte cell line (NHC) was isolated from a normal human liver and characterized by Joplin $e a^{17}$ and was a gift from Dr Juan Medina (University of Navarra, Pamplona, Spain). NHCs passages 10-15 were used in this study. We also used a cell line derived from a patient with PSC previously described by us. ${ }^{18}$ The human macrophage THP-1 cell line was purchased from the American Type Culture Collection (Manassas, VA) and cultured according to their protocol.

\section{Cholangioid Media}

Media previously reported for culturing cholangiocytes in monolayers (H69 media) ${ }^{19}$ was supplemented with $5 \mathrm{ng} / \mathrm{ml}$ of recombinant epidermal growth factor (EGF) (Sigma-Aldrich) and 5\% of growth factor reduced, phenol free Matrigel (Corning, New York, NY).

\section{D Cultures}

To prevent the growth of cells in monolayer, tissue culture dishes or glass slide chamber slides were pre-coated with a polyHEMA (Sigma-Aldrich, St Louis, MO) solution (125 $\mathrm{\mu l} /$ $\left.\mathrm{cm}^{2}\right) .{ }^{20}$ A cell suspension containing $\sim 6000$ NHCs or PSC cells and $56 \mu \mathrm{l}$ of growth factor reduced, phenol-free Matrigel/ $\mathrm{cm}^{2}$ was plated on the polyHEMA-coated surfaces and incubated at $37^{\circ} \mathrm{C}, 5 \% \mathrm{CO}_{2}$ for $2 \mathrm{~h}$ as reported elsewhere. ${ }^{21}$ Then, $250 \mu \mathrm{l}$ of cholangioid media were added per well. Cholangioid media was changed every 4 days and the cells kept in culture for 5-20 days.

\section{Isolation and Counting Cells of Cholangioids}

Five- and twenty-day single cholangioids of up to $150 \mu \mathrm{m}$ were isolated by manually pipetting using $10 \mu \mathrm{l}$ pipettes under a Zeiss Axiovert 25 microscope. The cholangioids were placed in $25 \mu \mathrm{l}$ of Dispase (Corning) and incubated at $37^{\circ} \mathrm{C}, 5 \% \mathrm{CO}_{2}$ for $2 \mathrm{~h}$ to dissociate the cells. Single cells were counted using a hemocytometer and Trypan blue. The cholangioid size was calculated by averaging the vertical, horizontal, and diagonal diameters of each cholangioid using the ImageJ software.

\section{Phase Contrast and Confocal Live Microscopy}

Pictures of five- and twenty-day cholangioids were taken via phase contrast, differential interference contrast (DIC), and confocal microscopy using a Nikon Eclipse TE 300 and a Zeiss LSM 780 microscopes.

\section{Immunofluorescent Microscopy}

Cholangioids were fixed with $4 \%$ paraformaldehyde, permeabilized for $45 \mathrm{~min}$ at $4{ }^{\circ} \mathrm{C},{ }^{21}$ blocked with $1 \%$ donkey serum, and incubated overnight at $4{ }^{\circ} \mathrm{C}$ with antibodies to the following proteins: GM130 (Novus Biologicals, Littleton, CO); SR, CK7, and CK19 (Santa Cruz Biotechnology); acetylated tubulin (Sigma-Aldrich); and IL-6, $\gamma$ H2A.x, CFTR, $\mathrm{AE} 2$, and CD11B (Abcam, Cambridge, MA). All antibodies were used at a concentration of 1:100. Following three washes, the cholangioids were incubated with the following secondary antibodies diluted at 1:400: donkey anti-goat IgG-568, donkey anti-rabbit IgG-488, and donkey antimouse-647 (Abcam). Then nuclei were counterstained with DAPI and the chamber slides were stored at $4{ }^{\circ} \mathrm{C}$ in $20 \%$ glycerol/phosphate-buffered saline (PBS).

\section{Transmission Electron Microscopy}

Twenty-day cholangioids were fixed in $2.5 \%$ glutaraldehyde/ $0.1 \mathrm{M}$ phosphate buffer $(\mathrm{PB})$ at $\mathrm{pH} 7.4$ for $1 \mathrm{~h}$ at room temperature. Then, the cholangioids were washed in $0.1 \mathrm{M} \mathrm{PB}$ $\left(\mathrm{pH}\right.$ 7.2-7.4) and incubated with $1 \% \mathrm{OsO}_{4}$ in $\mathrm{PB}$ for $1 \mathrm{~h}$. After rinsing with $\mathrm{PB}$ twice and four times with distilled water, the samples were dehydrated, and then embedded in Spurrs resin and sectioned at $90 \mathrm{~nm}$. Pictures were taken using a Joel 1400 electron microscope (Joel, Peabody, MA, USA). 


\section{Immunogold Transmission Electron Microscopy}

Pellets of exosomes (see below for isolation) were fixed in $4 \%$ paraformaldehyde/0.1 M PB (1:1 in volume) for $2 \mathrm{~h}$ or overnight at $4{ }^{\circ} \mathrm{C}$. The samples were then placed on Formvar-carbon- coated nickel grid (200 mesh) and air-dried for 30 min. After saturation of free aldehyde groups by $2 \%$ FCS/PBS (fetal calf serum), containing $20 \mathrm{mmol} / \mathrm{l}$ glycine for $20 \mathrm{~min}$ and blocked with $10 \% \mathrm{FCS} / \mathrm{PBS}$ for $20 \mathrm{~min}$, the grids were incubated with a

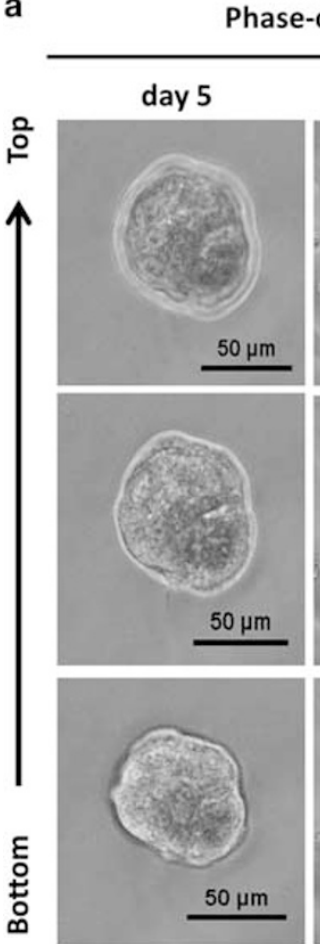

Phase-contrast
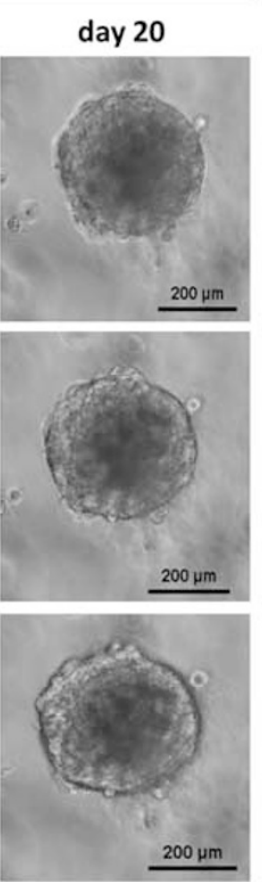

DIC
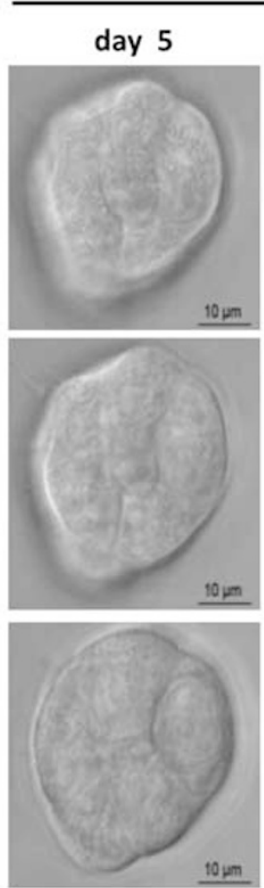
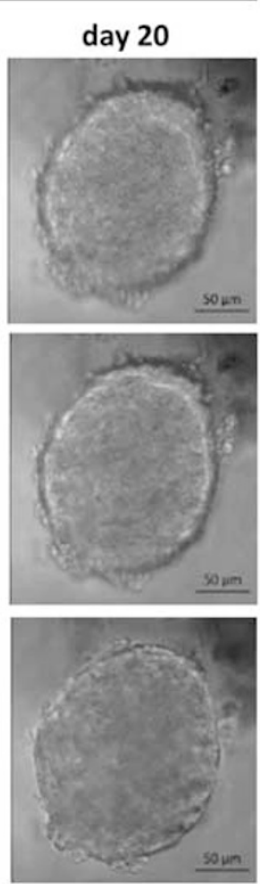

b
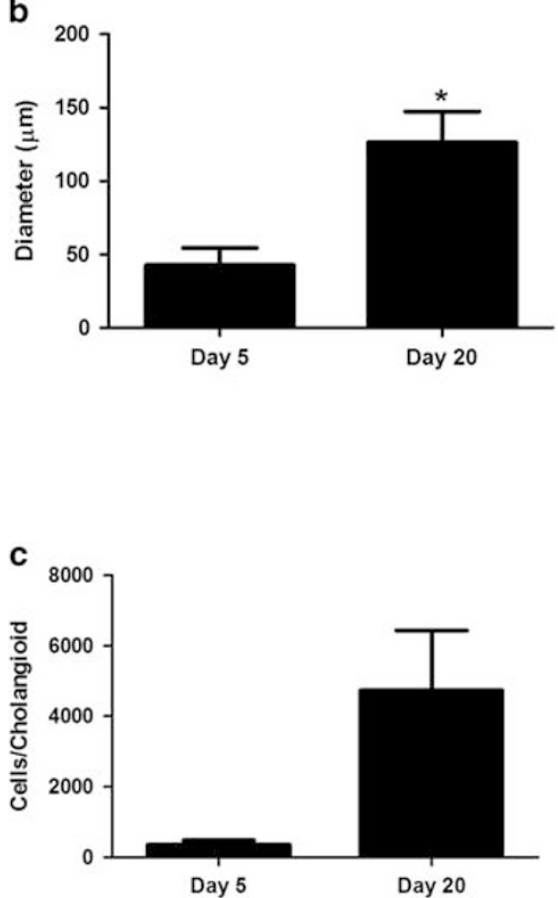

Figure 1 3D formation of cholangioids. (a) Live images of 5-day and 20-day 3D cultures of NHCs in Matrigel. (b) Pictures were taken using phase contrast $(\times 20)$ or DIC ( $\times 63)$ microscopy. (c) Individual cholangioids were imaged and isolated after days 5 and 20 in culture. The cholangioids were disassembled into individual cells, which were counted using trypan blue, $n=3, * P<0.05$. Diameter measurements were taken on the pictures of the isolated 5- and 20-day cholangioids using ImageJ. The data shown are representative of triplicate experiments, ${ }^{*} P<0.05$.

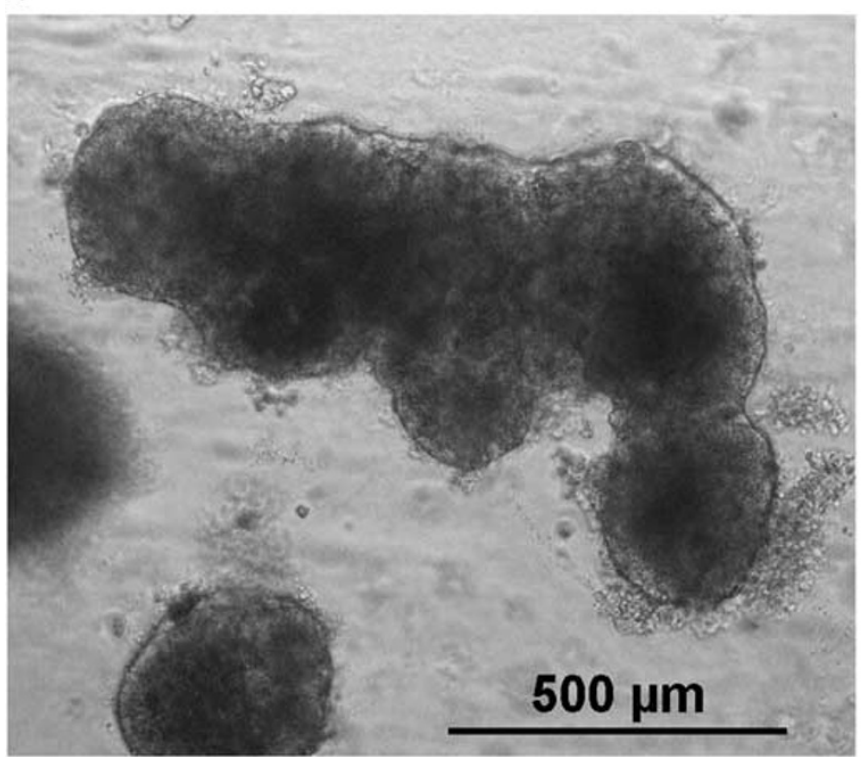

b

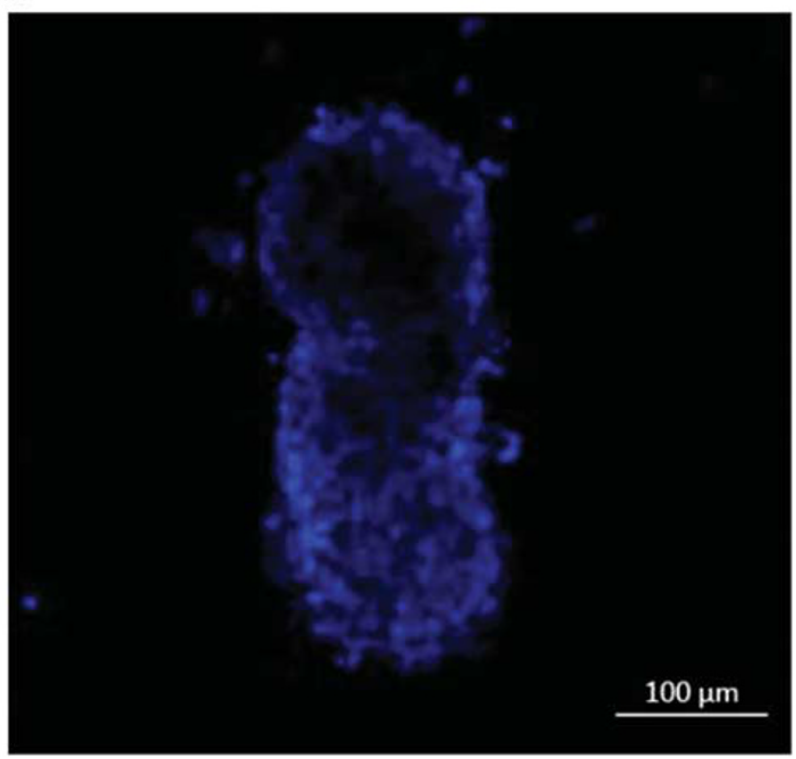

Figure 2 Tubular-like structure formation from NHC cholangioids. (a) NHCs were cultured in Matrigel for 20 days to form cholangioids. Tubular-like structures were also formed as shown by bright field $(\times 20)(\mathbf{b})$ and confocal $(\times 63)$ microscopy, blue represents nuclei stained with DAPI. 

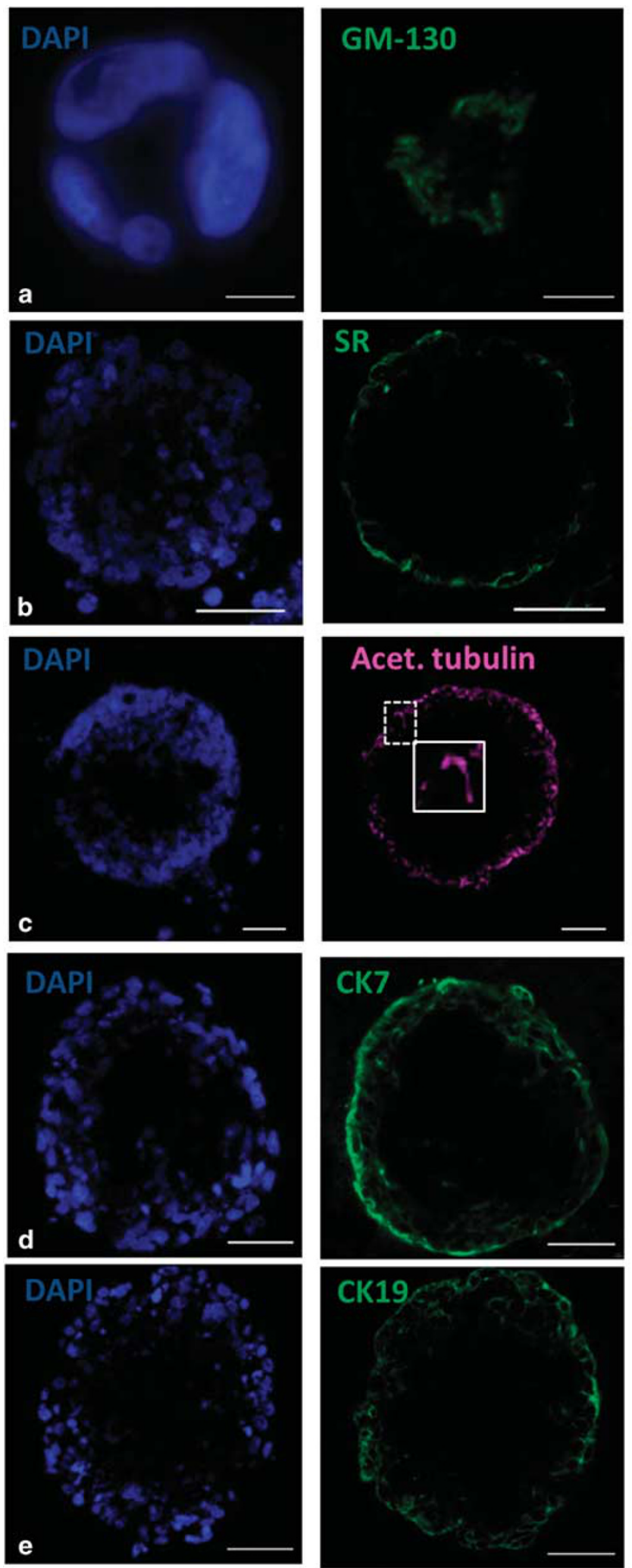
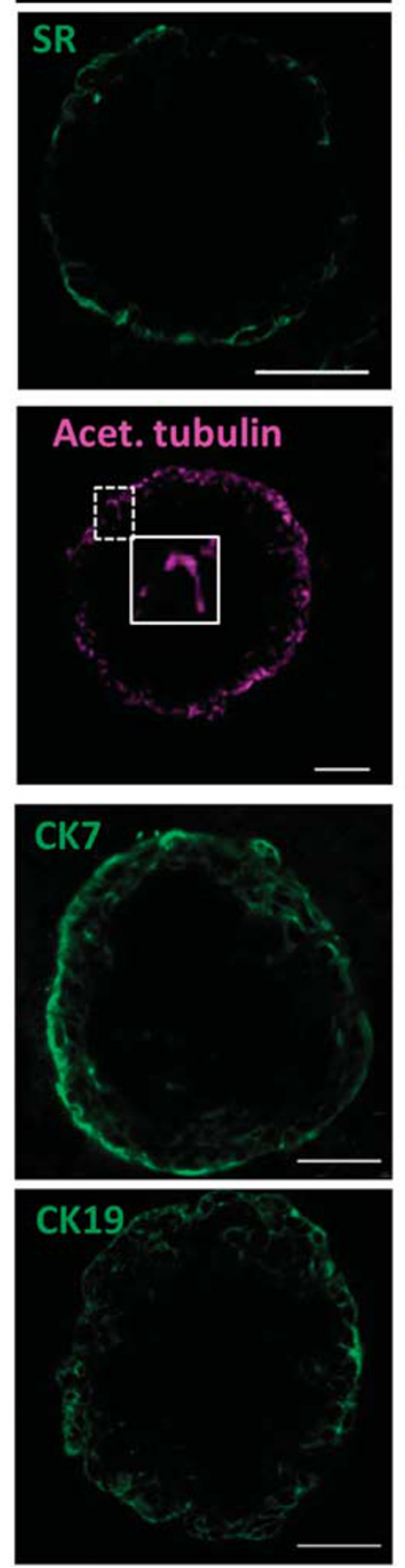
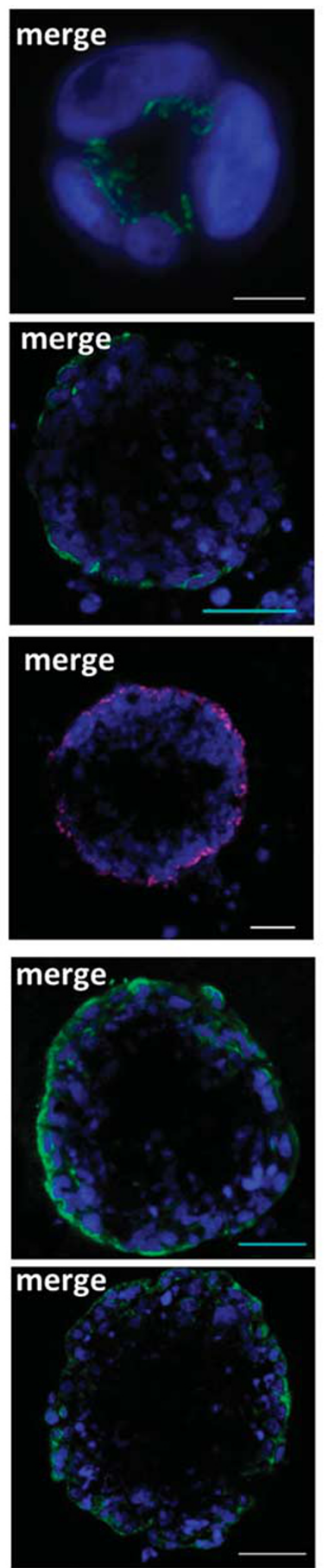

Figure $3 \mathrm{NHC}$ cholangioids are polarized and retain cholangiocyte markers. (a) Immunofluorescence of the polarity markers GM130 on the apical side, scale $b a r=10 \mu \mathrm{m}$, (b) the secretin receptor (SR) located on the basolateral membrane, scale bar $=50 \mu \mathrm{m}$, (c) acetylated tubulin on the apical side extending into the lumen, insert showing an enlarged picture of a primary cilium, scale bar $=50 \mu \mathrm{m}$, (d) the cholangiocyte markers CK7, scale bar $=50 \mu \mathrm{m}$, (e) and CK19, scale bar $=50 \mu \mathrm{m}$. (f) Western blot of the cholangiocyte markers GGT1, CK7, and CK19 and Actin from cholangioid lysates. 
TSG101 antibody (Santa Cruz, goat M-19) diluted 1:20 with $10 \% \mathrm{FCS} / \mathrm{PBS}$ for $2 \mathrm{~h}$ or overnight at $4{ }^{\circ} \mathrm{C}$. After washing with $2 \% \mathrm{FCS} / \mathrm{PBS}$ six times ( 5 min each) and labeling with rabbit anti-goat IgG (Sigma) in 1:500 dilution with $10 \%$ FCS/PBS for $1 \mathrm{~h}$, the grids were labeled with protein A-10 nm gold (1:20 diluted with $10 \% \mathrm{FCS} / \mathrm{PBS}$ ). The grids were post fixed with $1 \%$ gutaraldehyde/PBS for $5 \mathrm{~min}$, washed six times with distilled water drops ( 3 min each). Then, the grids were contrasted and embedded with a mixture of $4 \%$ uranyl acetate and $2 \%$ methylcellulose (in 1:9 ratio). The grids were airdried and observed with JEOL 1400 electron microscope.

\section{Western Blotting}

Approximately, $5-10 \mu \mathrm{g}$ of protein were run on $4-20 \%$ Mini-PROTEANTGX precast polyacrylamide gels (Bio-Rad Laboratories, Hercules, CA), transferred to nitrocellulose membranes, blocked, and probed with primary antibodies for: CD63, ALIX, TSG101, Actin, CK7, CK19, and GGT1 diluted 1:500 (Santa Cruz Biotechnology). The secondary antibody, donkey-anti-rabbit or mouse-HRP (Jackson Labs, West Grove, PA) was diluted 1:10000. The signal was developed with the ECL Western Blotting System (GE Healthcare, Little Chalfont, UK) using a Kodak X-Omat (Rochester, NY).

\section{RNA Extraction and Quantitative Real-Time PCR}

Twenty-day cholangioids from NHC-control and NHC-sen were collected using Cell Recovery Solution (Corning, Corning, NY) according to the manufacturer's protocol. Total RNA was extracted from cholangioids using the RNeasy Plus Mini Kit (Qiagen). Reverse transcription was performed with $1 \mu \mathrm{g}$ RNA, using oligo (dT) primer and SuperScript III. Real-time PCR was performed in a volume of $20 \mu \mathrm{l}$ using Sybr Green Master Mix and QuantStudio3 (Applied Biosystems).
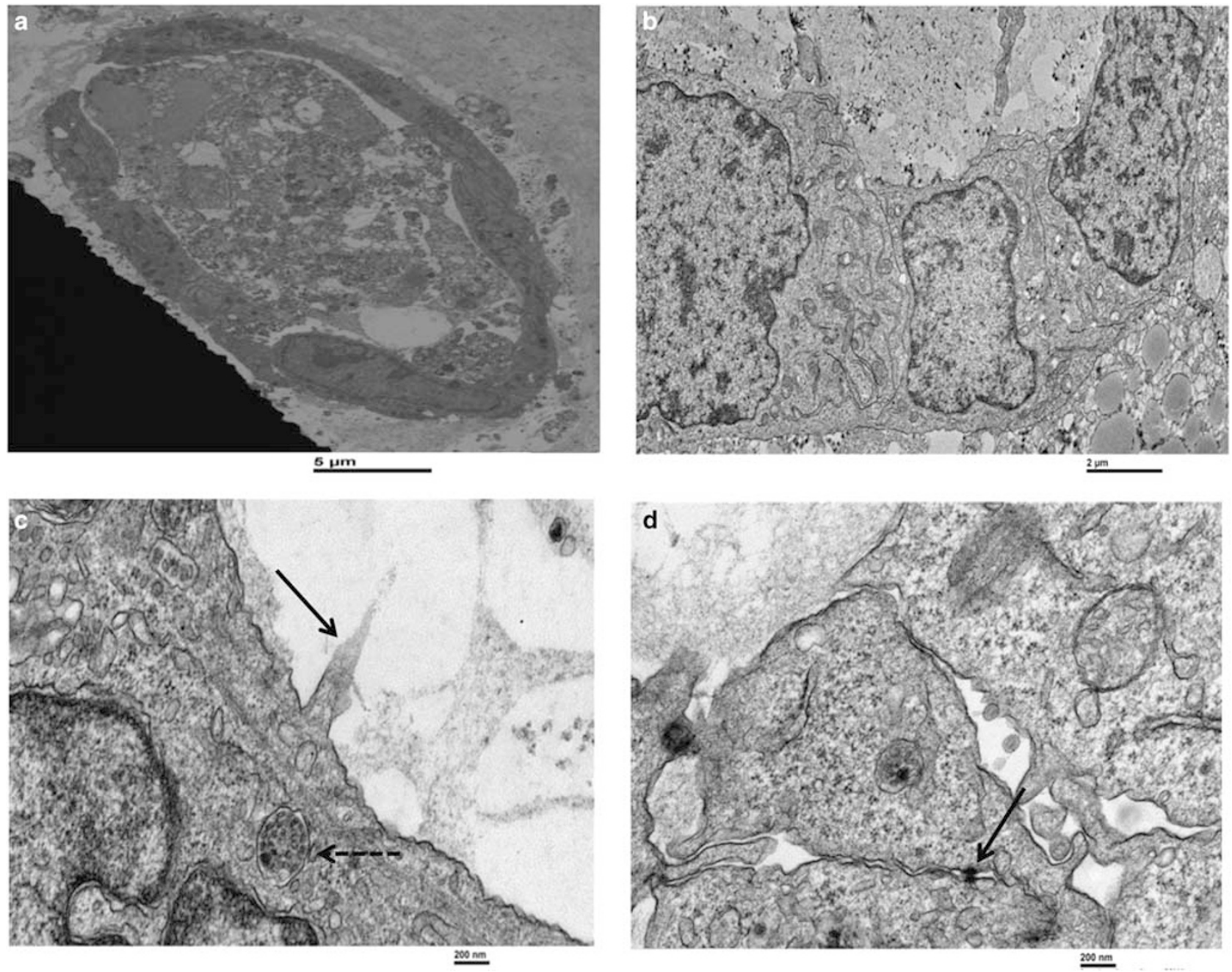

Figure 4 The ultrastructure of NHC cholangioids via electron microscopy. NHCs were cultured in Matrigel for 20 days to form cholangioids before fixation and processing for electron microscopy. (a) Cross-section of a cholangioid showing a layer of live cells with apical and basolateral polarity and a luminal area formed by residual of dead cells. (b) Higher magnification showing the cytoskeleton of NHCs. (c) Arrow pointing at partial cilium extending into the lumen and dashed arrow showing a multivesicular body. (d) Arrow pointing at tight junctions between NHCs. 


\section{Exosome Isolation}

Twenty-day cholangioids were washed in serum-free H69 media three times. Then, the cholangioids were incubated in serum free $\mathrm{H} 69$ media for $24 \mathrm{~h}$ at $37^{\circ} \mathrm{C}, 5 \% \mathrm{CO}_{2}$. The conditioned media was collected and processed for differential ultracentrifugation to isolate exosomes as described previously. ${ }^{22}$

\section{Secretin Assays}

Twenty-day cholangioids were incubated with vehicle or $100 \mathrm{nM}$ recombinant human secretin (Sigma-Aldrich) and stained with 1:1000 Cell MaskOrange Plasma membrane stain (Life Technologies, Carlsbad, CA) for $15 \mathrm{~min}$ at $37^{\circ} \mathrm{C}, 5 \%$ $\mathrm{CO}_{2}$. After two washes with cholangioid media live images where taken using a Zeiss LSM 510 microscope.

\section{Experimentally Induced Senescence of Cholangioids}

To induce senescence, NHCs were cultured in Matrigel for 20 days as described earlier to generate cholangioids. The next day, after plating the $3 \mathrm{D}$ cultures, the cholangioids were treated with $50 \mathrm{nM} \mathrm{H}_{2} \mathrm{O}_{2}$ every other day. ${ }^{2}$

\section{$\beta$-gal Assays}

For the colorimetric $\beta$-gal assays, 20-day cholangioids were washed three times with PBS and were fixed for $15 \mathrm{~min}$ at room temperature. Following three washes with PBS, the cholangioids were incubated with the $\mathrm{X}$-gal solution for $4 \mathrm{~h}$ at $37^{\circ} \mathrm{C}, 5 \% \mathrm{CO}_{2}$ (Cell Biolabs, San Diego, CA). The cholangioids were washed three times with PBS before adding 20\% glycerol/ PBS. The samples were stored at $4{ }^{\circ} \mathrm{C}$ before taking pictures at $\times 20$ with a Nikon Eclipse TE 300. For the quantitative assay, a fluorescence $\beta$-gal kit was used. The cholangioids were washed and incubated with the SA- $\beta$-gal substrate according to the manufacturer's instructions. Fluorescence was read using a Synergy H1 microplate reader, Biotek.

\section{Freezing Cholangioids and Culture of Frozen Cholangioid Stocks}

Media was pipetted out from 20-day cholangioids that were grown in $35 \mathrm{~mm}$ dishes. Approximately $1 \mathrm{ml}$ of cold cholangioid media was added to the dish placed on ice. The media was pipetted up and down several times until the Matrigel was dissolved and transferred to a conical tube and spun for $3 \mathrm{~min}, 4^{\circ} \mathrm{C}, 1500$ r.p.m. The media was aspirated and the pellet resuspended in cold cholangioid media containing 10\% DMS0 and 10\% growth factor reduced, phenol-free Matrigel and stocks prepared in cryovials. The cryovials were placed at $-80^{\circ} \mathrm{C}$ for $24 \mathrm{~h}$, and then stored in liquid $\mathrm{N}_{2}$. To culture frozen cholangioid stocks, the cryovial was placed in a water bath at $37^{\circ} \mathrm{C}$ for $1 \mathrm{~min}$. The cholangioid suspension was mixed with cold cholangioid media and spun for $3 \mathrm{~min}$ at $4{ }^{\circ} \mathrm{C}, 1500$ r.p.m. After aspirating the media, the tube was placed in ice and the pellet resuspended in $450 \mu \mathrm{l}$ of growth factor reduced, phenol-free Matrigel. Then the cholangioid-Matrigel suspension was placed on a polyHEMAcoated $35 \mathrm{~mm}$ dish as described earlier.

\section{Co-Cultures}

Approximately 10000 THP-1 cells in suspension were incubated with 20-day-old NHC and PSC cholangioids cultured in glass slide eight-well chamber slides for $24 \mathrm{~h}$ at $37^{\circ} \mathrm{C}, 5 \% \mathrm{CO}_{2}$ in cholangioid media. Then the co-cultures were processed for live images or immunofluorescence.

\section{Cell Migration Assay}

Human monocytic THP-1 cells (TIB-202, ATCC) were plated at 200000 cells/well in the upper chamber of a 96-well migration assay (Cell Biolabs, San Diego, CA) and incubated for $6 \mathrm{~h}$ with conditioned media (bottom chamber) obtained from NHC-Ctrl or NHC-sen organoids or with media only as a negative control. THP-1 cell migration was then quantitated per manufacturer's instructions.

\section{Statistical Analysis}

The data reported represents the mean of 5-12 cholangioids per experiment or fold change \pm standard error of the mean. The results were analyzed using Graphpad Prism by two-tailed, paired or unpaired $t$-test. $P$-values of $<0.05$ were considered statistically significant.
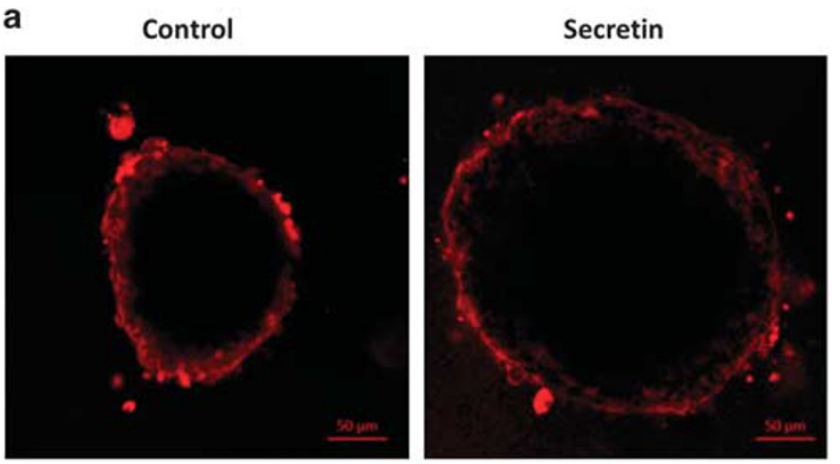

b

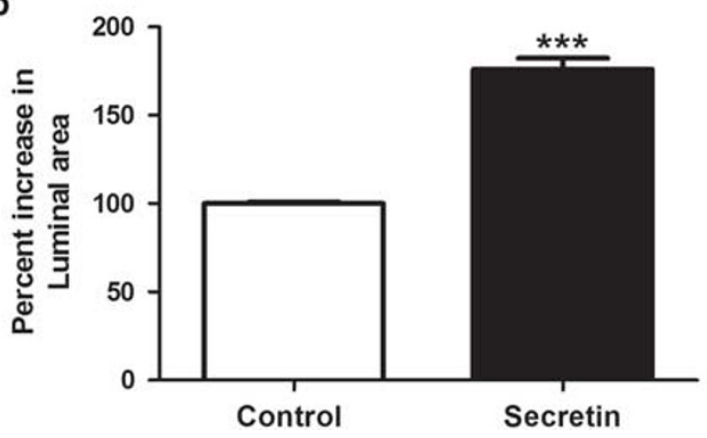

Figure 5 Secretin induces NHC-cholangioid size. (a) NHCs were cultured for 20 days in Matrigel to form cholangioids. Cholangioids were treated with vehicle or $100 \mathrm{nM}$ human recombinant secretin for $15 \mathrm{~min}$ and CellMask Orange Plasma membrane stain 1:1000. After two washes with cholangioid media, live images were taken at $\times 63$ using confocal microscopy. (b) Average of measurements of vertical, horizontal, and diagonal diameters taken using ImageJ were recorded. The data shown are representative of triplicate experiments. ${ }^{*} P<0.01$. 


\section{RESULTS}

\section{Development and Characterization of Cholangioids}

Live images of 5- and 20-day cholangioids using phase contrast and DIC microscopy (Figure 1a) showed that, in Matrigel, NHCs form spheroid-like structures that increase in size (Figure 1b) and cell number (Figure 1c). After 20 days in culture, cholangioids start forming tubular structures resembling bile ducts (Figures 2a and b). Immunofluorescence analysis showed that cholangioids are polarized structures demonstrated by the expression of the Golgi protein, GM130, that has been used as an apical marker in $3 \mathrm{D}$ cultures as it shows the localization of the Golgi apparatus toward the lumen of hollow spheroids, ${ }^{21}$ as well as the secretin receptor (SR) on the basolateral domain of cholangioids (Figures 3a and b). Immunofluorescence and electron microscopy showed that cholangioids also possess primary cilia that extend into a hollow lumen (Figures $3 \mathrm{c}$ and $4 \mathrm{c}$ ). Moreover, studies performed with 3D structures of other cell types showed that spheroids are hollow structures and that their lumens are formed as the cells in the middle die via apoptosis and autophagy-dependent mechanisms. ${ }^{21,23,24}$ Electron microscopy analysis shows the luminal space and remnants of dead cells of a cholangioid and the ultrastructure of the NHCs forming cholangioids depicts the cytoskeleton, nucleus, a multivesicular body, and tight junctions between cells as well (Figures 4a, b, and d). A 3D reconstruction of the z-stacks taken via confocal microscopy confirms that indeed cholangioids have a hollow internal structure (Supplementary Figure 1). Moreover, NHCs forming cholangioids retain expression of the cholangiocyte markers CK7, CK19, and
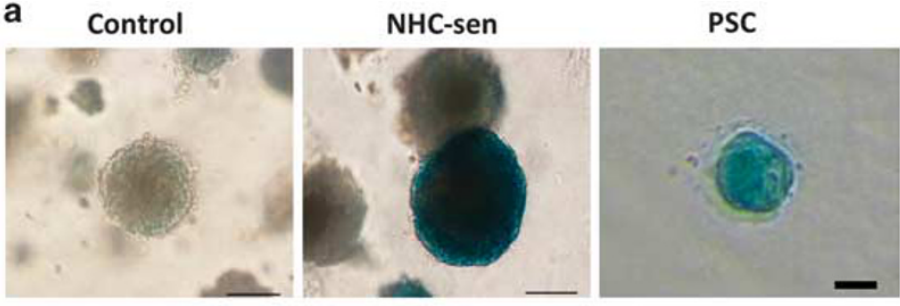

b

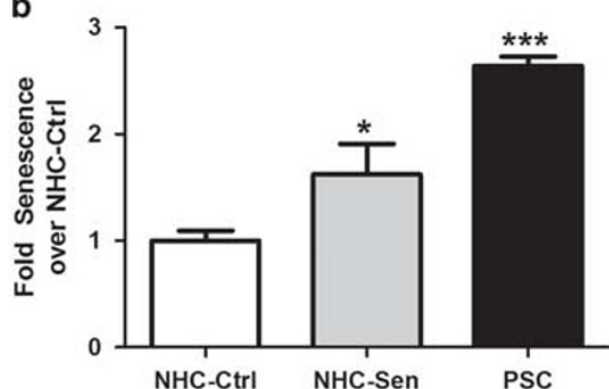

C
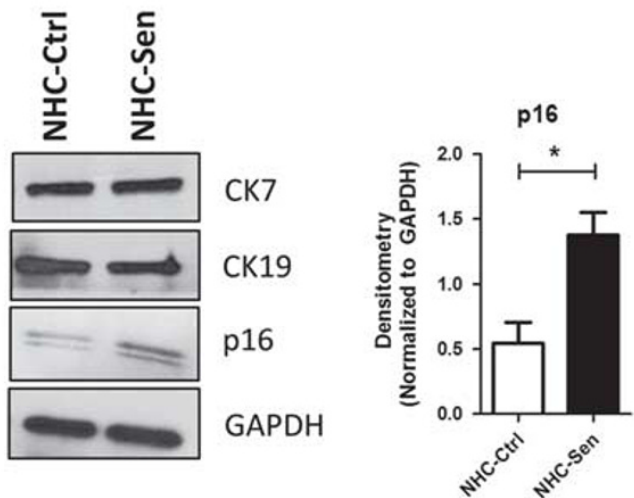

d
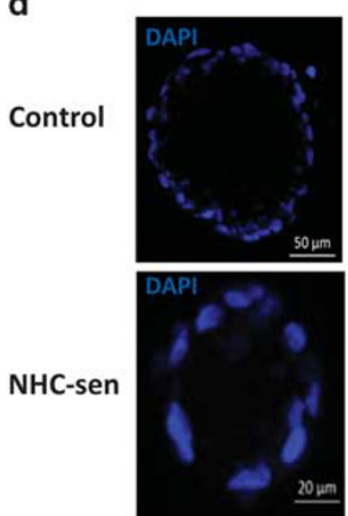
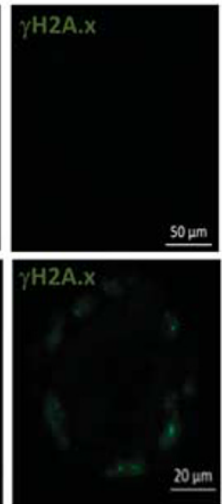
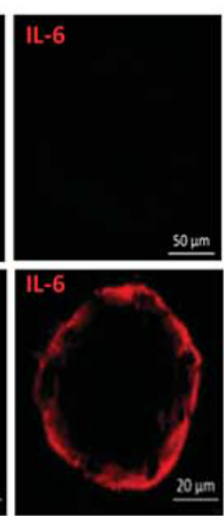

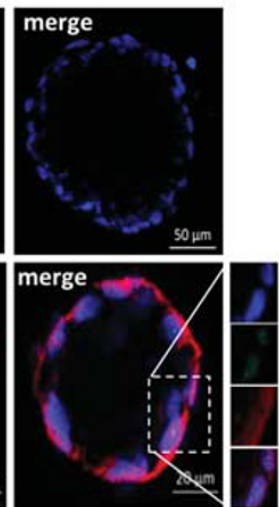

e

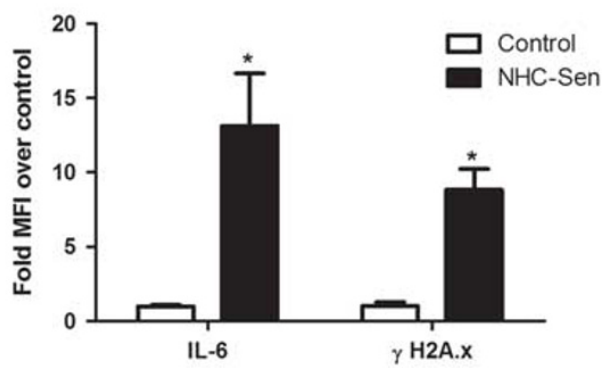

Figure $6 \mathrm{H}_{2} \mathrm{O}_{2}$ induces NHC-cholangioid senescence. NHCs or PSC cells were grown in Matrigel for 20 days to form cholangioids. (a) Control, NHC-sen, and PSC cholangioids were processed for a qualitative $\beta$-gal assay and bright-field pictures. Blue color represents senescent cholangioids. Control and NHC-sen, scale bars $=100 \mu \mathrm{m}$, PSC scale bar $=50 \mu \mathrm{m}$, and (b) a quantitative fluorescent $\beta$-gal assay showing fold senescence over control cholangioids is depicted, $n=3,{ }^{*} P<0.05$. (c) Western blot of CK7, CK19, and p16 from NHC-control, NHC-sen (left), densitometry for p16 (right). (d) Control and NHC-sen cholangioids were fixed and processed for immunofluorescence and stained for the senescent and SASP markers $\gamma \mathrm{H} 2 \mathrm{~A} . \mathrm{x}$ and IL-6, respectively. Insert showing an enlarged picture of the punctuated pattern of $\gamma \mathrm{H} 2 \mathrm{~A} . \mathrm{x}$ and IL- 6 staining. (e) Mean fluorescence intensity of $\gamma \mathrm{H} 2 \mathrm{~A} . \mathrm{x}$ and IL-6 was measured using ImageJ and fold-change over control was calculated. The data shown are representative of triplicate experiments. ${ }^{*} P<0.01$. 
GGT1 (Figures 3d-f). Immunofluorescence also showed that cholangioids express CFTR, a cell surface chloride transporter, as well as the anion exchanger, AE2 (Supplementary Figure 2). We were also able to make stocks of frozen NHC cholangioids, store them for 8 months in liquid $\mathrm{N}_{2}$, re-culture them, and show that they maintain their morphology and cholangiocyte marker expression (Supplementary Figure 3).

\section{Functional Features}

As cholangiocytes are known to respond to secretin, we stimulated cholangioids with recombinant human secretin to test whether cholangioids were functionally active. We found that secretin induced a $75.9 \pm 6.4 \%$ increase $(P<0.01)$ in the cholangioid luminal area (Figure 5) showing that NHCderived cholangioids are responsive to secretin and are functionally active.

\section{Pathophysiology}

Our previous studies indicated that cholangiocyte senescence is an important cellular phenotype in PSC and that cholangiocytes in liver biopsies from PSC patients express increased levels of the SASP pro-inflammatory factors IL-6, IL-8, CCL2, and PAI- $1 .^{2}$ We also reported that cholangiocytes isolated from PSC patients show characteristics of cellular senescence, ie, SA- $\beta$-gal expression as well as markers of SASP, such as, IL-6, IL-8, and CCL2 expression. ${ }^{18}$ Furthermore, previously we were able to induce senescence of NHCs on cholangiocyte monolayers using hydrogen peroxide $\left(\mathrm{H}_{2} \mathrm{O}_{2}\right)^{2}$ and we were able to reproduce that outcome here in cholangioids (Figure 6). In addition, we were also able to recapitulate the senescence phenotype in our 3D model by using a cholangiocyte cell line from a patient with PSC that was previously isolated and characterized by our group. ${ }^{18} \mathrm{We}$ confirmed senescence using a qualitative SA- $\beta$-gal assay as shown on Figure 6a. A quantitative SA- $\beta$-gal technique demonstrated $1.62 \pm 0.02$ - and 2.64 \pm 0.09 -fold increase senescence over control for the NHC-sen and PSC cholangioids, respectively $(P<0.02)$ (Figure $6 \mathrm{~b})$. Western blotting analysis depicted an increase in the senescence marker p-16 with the $\mathrm{H}_{2} \mathrm{O}_{2}$ treatment and in PSC cholangioids (Figure $6 \mathrm{c}$ ), consistent with the previous reports from our group. We also detected the expression of the senescent marker $\gamma \mathrm{H} 2 \mathrm{~A} . \mathrm{x}$ and
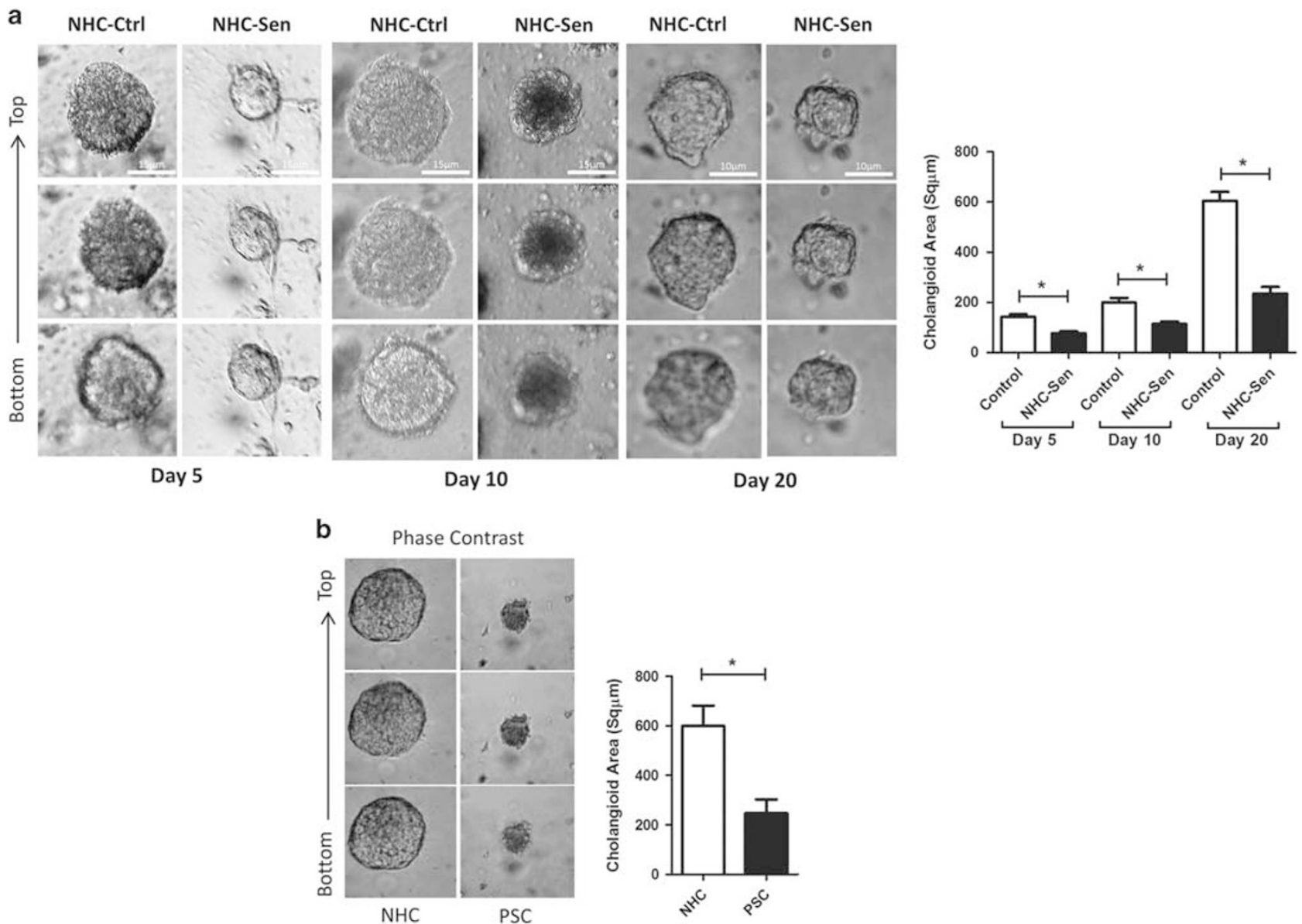

Figure 7 Senescent cholangioids display smaller diameter and reduced growth rate. (a) Quantification of phase contrast from NHC-control and NHC-sen during 5,10 , and 20 days of 3D culture, showed that NHC-sen generate smaller cholangioids and a slower growth rate compared to NHC-control. (b) A similar size reduction was observed in PSC cholangioids after 20 days in 3D culture. 
a

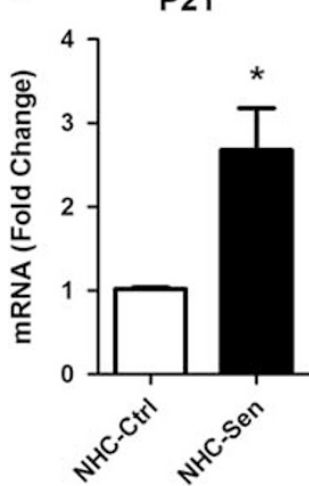

b

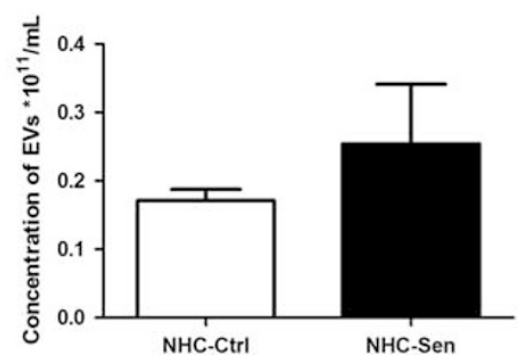

CCL2
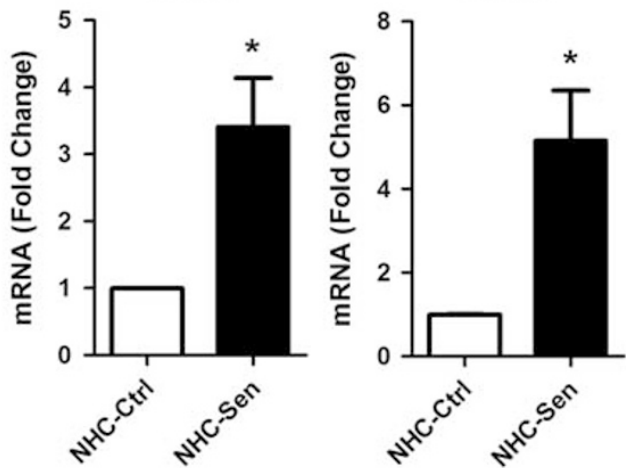

C

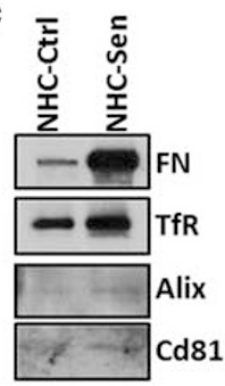

IL-8
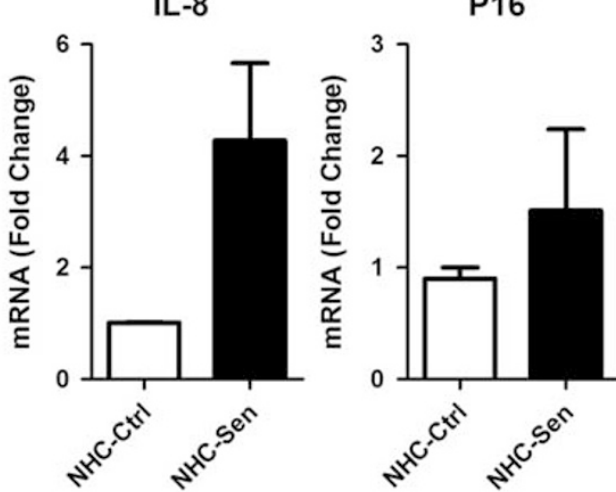

d

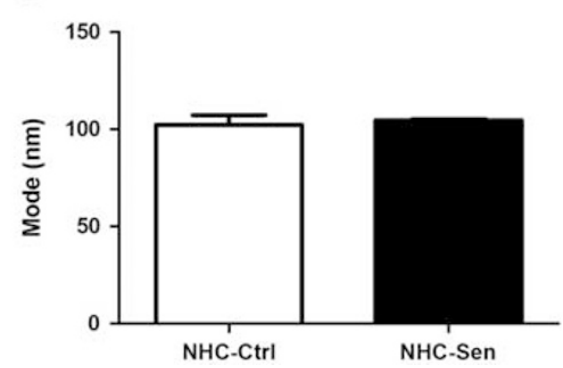

Figure 8 NHC-sen cholangioids are secretory. (a) Real-time PCR showed an enrichment of senescent markers (P21, B-Gal, CCL2, IL-8, and P16) in NHCsen cholangioids when compared to NHC-control cholangioids. (b) Media from 21-day cholangioids was collected and processed for exosome isolation. Nanoparticle tracking analysis showing an increased concentration (particles/ml) in NHC-sen. (c) Western blot of the exosomal markers Alix, CD68, and TSG101 from exosomes released by cholangioids. (d) Nanoparticle tracking analysis showing the size of exosome isolation from cholangioids. ${ }^{* *} P \leq 0.05$. All PCR data are normalized to GAPDH and NHC-control cholangioid expression is set to 1.0. The data shown are representative of triplicate experiments. ${ }^{*} P<0.01$.

the SASP marker IL-6 via immunofluorescence (Figure 6d) and found that the mean fluorescence intensity of the NHCsen cholangioids was upregulated $8.8 \pm 1.4$ - and $12.7 \pm 3.4$ fold for $\gamma$ H2A.x and IL-6, respectively, over the control cholangioids $(P<0.01)$ (Figure 6e). Interestingly, we observed that $41 \pm 16 \%$ of the NHC-sen cholangioids and virtually all of the PSC cholangioids failed to form lumens; in contrast all the NHC cholangioids formed lumens. Quantitative phase contrast imaging showed that NHC-sen cholangioids displayed a smaller diameter and a slower rate of growth as they progressed from day 5 to day 20 (Figure 7a). A similar size reduction was observed in PSC cholangioids as well (Figure 7b). Unlike the NHC cholangioids, the PSC cholangioids did not exhibit polarized distribution of GM130, and the SR and secretin stimulation did not result in an increase in PSC cholangioid size (data not shown). Our lab has previously reported that senescent cholangiocytes become highly secretory including the release of extracellular vesicles (EVs) or exosomes. Here we show that the senescent cholangioids also become highly secretory. Based on Q-PCR studies, the senescent cholangioids upregulate typical markers of senescence such as P21, $\beta \mathrm{Gal}$, and p16 and generate increased levels of pro-inflammatory cytokines such as CCL2 and IL-8 (Figure 8a). Consistent with this secretory phenotype, senescent cholangioids also release an increased number of EVs (Figure 8b). These EVs, when characterized by immunoblotting, are positive for exosomal markers such as FN, Alix, TfR, and Cd81 (Figure 8c). Nanotracking analysis (NTA) also shows the size of these EVs to be around $106 \mathrm{~nm}$, consistent with the size of exosomes (Figure $8 \mathrm{~d}$ ). Due to the limited availability of PSC cells, we were not able to generate PSC cholangioids to isolate exosomes.

Next, to test whether we could recapitulate the macrophage accumulation phenotype of $\mathrm{PSC}^{3}$ in our model, we cocultured normal and PSC cholangioids with a commonly used human macrophage cell line, THP-1.25,26 After $24 \mathrm{~h}$ in culture, the co-cultures were fixed and stained for CK7 and $\mathrm{CD} 11 \mathrm{~B}$, the former a marker for cholangiocytes and the latter a marker of macrophage activation (Figure 9a). The number of THP- 1 cells associated with PSC cholangioids was $7.6 \pm 1.6$ $(P<0.03)$ compared to $1.3 \pm 0.6$ for the control cholangioids (Figure 9b). Furthermore, conditioned media experiments revealed that cell-free media derived from NHC-sen cholangioids resulted in a two-fold increase in the migration of THP-1 cells, compared to media derived from NHC-Ctrl cholangioids (Figure 9c).

\section{DISCUSSION}

In this study, we describe the establishment and characterization of cholangioids, a novel, 3D model of cultured cholangiocytes 
a

THP-1 (CD11b)
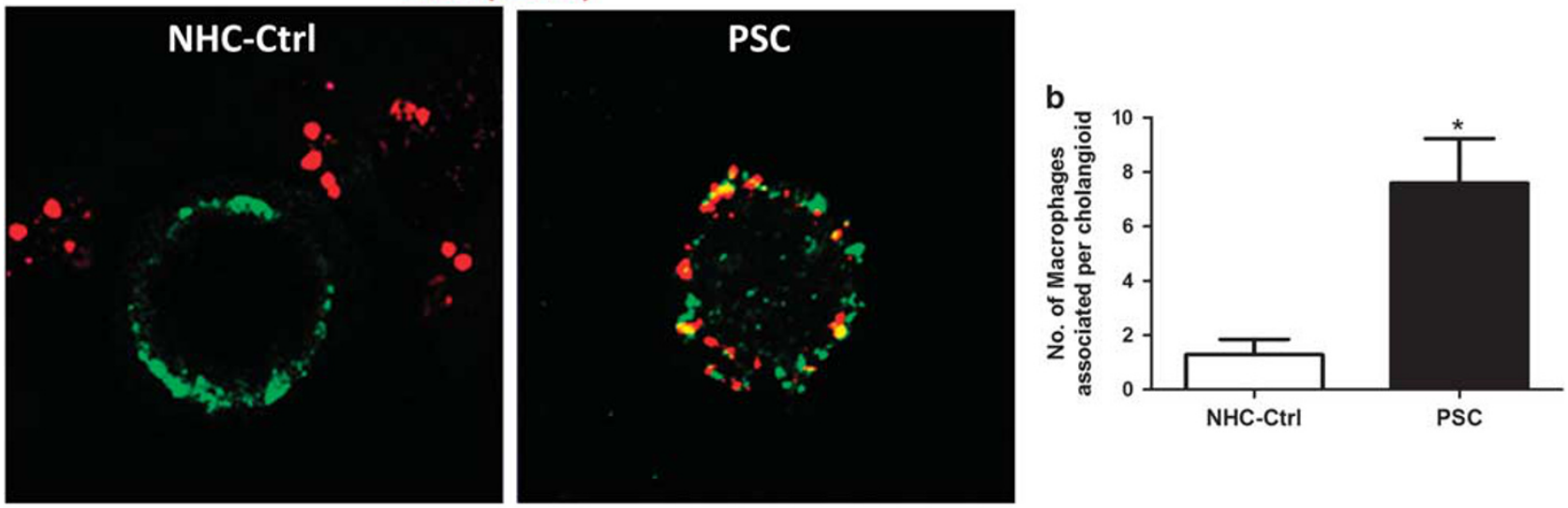

C

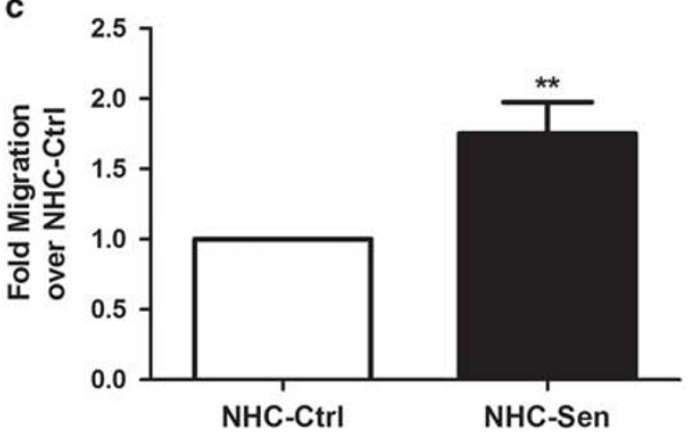

Figure 9 Co-culture of THP-1 cells with NHC and PSC cholangioids. (a) NHC and PSC cholangioids were co-cultured with THP-1 cells for 24 h. The cocultures were fixed and stained for CK7 in green and CD11B in red. (b) The number of THP-1 cells associated per cholangioid was counted and plotted in a bar graph. (c) THP-1 cells were exposed to conditioned media from NHC or NHC-sen and subjected to a migration assay. The data shown are representative of triplicate experiments. ${ }^{*} P<0.005$.

to study the pathogenesis of PSC. Beginning with cholangiocyte cell lines, we generated $3 \mathrm{D}$ spheroids that resemble bile ducts, describing them morphologically and biochemically, and demonstrating that these cholangioids have functional features of both normal and senescent cholangiocytes. The data support the utility of this in vitro model to study the pathogenesis of PSC and perhaps other cholangiopathies. Also, to our knowledge, this is the first report describing isolation of exosomes from normal and senescent human cholangiocytes in $3 \mathrm{D}$ culture. The SASP is a characteristic feature of senescent cells and is increasingly thought to play a pathophysiologic role in biliary disease. ${ }^{2}$ Given that cellular senescence is a prominent feature of the cholangiocytes from patients with PSC, generating a 3D culture model that maintains the functional features of senescence and the SASP represents an important advance.

The method we describe in this manuscript was a modified version of the protocol described by Debnath et al. ${ }^{21}$ The advantages of using this method were that (i) we could monitor the growth of the cholangioids via bright-field microscopy very easily; (ii) the growth of the cholangioids was consistent across different experiments; (iii) live images of the cholangioids interacting with macrophages using confocal microscopy could be performed and these same samples could then be fixed for immunofluorescence analysis; and (iv) the immunofluorescence technique in this protocol is very simple. As we cultured our cholangioids for 20 days, our protocol required the use of undiluted Matrigel, as diluting it compromises the formation of the 3D structure. The ability of cholangioids to form lumens allows manipulation of lumen size, as we did with secretin, an assay that cannot be performed on cells cultured on plastic. Furthermore, interactions between cells and their extracellular microenvironment and changes in the stiffness of the extracellular microenvironment are better visualized in a $3 \mathrm{D}$ compared to a $2 \mathrm{D}$ model. Thus, our $3 \mathrm{D}$ in vitro model has several advantages compared to cells cultured on plastic.

Several groups have shown that cholangiocytes form spheroids with a lumen in $3 \mathrm{D}$ culture, ${ }^{10,27-30}$ however this is the first report showing that senescent cholangioids, both experimentally induced or derived from PSC cholangiocytes, lack a lumen. The failure to form lumens could be a hallmark of PSC disease, as half of our NHC-sen and all of our PSC cholangioids did not form lumens. The reasons for this consistent observation remain unclear at this time. However, Tanimizu et $a^{28}$ showed that organoids that failed to form a luminal space were weakly positive for CK19 and had high expression of albumin. Another group also showed that 
albumin-/CK19+ cells form more and larger organoids when compared to albumin+/CK19 - organoids. ${ }^{31}$ Overall, this suggests that lumens are better-formed in organoids that highly or exclusively express cholangiocyte markers with a relative lack of hepatocyte markers. Western blot analysis of NHC-control and NHC-sen cholangioids showed similar levels of CK7 and CK19 expression (Figure 6c), and both cholangioids lack expression of albumin (data not shown). We have, at this point, no mechanistic explanation for why NHC-sen and PSC cells lack a central lumen, but we speculate that the percentage of cells in each of these cholangioid subtypes that were senescent may contribute to the differences. Although senescence is defined as terminal growth arrest, it is notable that the senescent and PSC cholangioids were still able to form cholangioids. We believe that the explanation for this is that the cells in this system are not uniformly senescent. The ability to expand and form cholangioids likely depends primarily on the non-senescent components of this model. Consistent with this, senescent and PSC cholangioids are smaller and have a slower growth rate than the corresponding controls, as would be expected if a proportion of the cells are senescent.

Macrophages are important cells of the immune system that are mobilized in response to an infection or accumulating damaged cells. The immunopathogenesis of PSC appears to involve the activation and recruiting of the innate immune system. ${ }^{3}$ Here we showed that our NHC-sen cholangioids retain the expression of profibroinflammatory SASP components. ${ }^{2,18}$ Co-culture of macrophages with cholangioids demonstrated that macrophages associate with and appear to be intercalated within the PSC cholangioid walls. The mechanisms of this phenomenon and the consequences of this cell-cell interaction remain to be determined, and our model provides an ideal system to interrogate this observation further.

In conclusion, studies on the pathogenesis of PSC have been hindered by the lack of a reliable $3 \mathrm{D}$ in vitro model of this disease. The model presented in this work recapitulates the senescence and SASP as well as the macrophage recruitment observed in PSC. The mechanisms by which macrophages are recruited to PSC can be studied with this model, and the broader question of how activated cholangiocytes interact with cells in the peribiliary extracellular matrix can be explored.

Supplementary Information accompanies the paper on the Laboratory Investigation website (http://www.laboratoryinvestigation.org)

\section{ACKNOWLEDGMENTS}

This work was supported by National Institutes of Health Grants DK57993 (NFL), DK41876 (GJG), DK100575 (RCH), the Satter Family Foundation, the Mayo Foundation, the Optical Microscopy Core of the Mayo Clinic Center for Cell Signaling in Gastroenterology (P30DK084567), and the Chris M Carlos and Catharine Nicole Jockisch Carlos Endowment Fund in Primary Sclerosing Cholangitis (PSC).

\section{DISCLOSURE/CONFLICT OF INTEREST}

The authors declare no conflict of interest.

1. Lazaridis KN, LaRusso NF. Primary sclerosing cholangitis. N Engl J Med 2016;375:1161-1170.

2. Tabibian JH, O'Hara SP, Splinter PL, et al. Cholangiocyte senescence by way of $\mathrm{N}$-ras activation is a characteristic of primary sclerosing cholangitis. Hepatology 2014;59:2263-2275.

3. Cameron RG, Blendis LM, Neuman MG. Accumulation of macrophages in primary sclerosing cholangitis. Clin Biochem 2001;34:195-201.

4. Solon J, Levental I, Sengupta K, et al. Fibroblast adaptation and stiffness matching to soft elastic substrates. Biophys J 2007;93: 4453-4461.

5. Antoni $\mathrm{D}$, Burckel $\mathrm{H}$, Josset $\mathrm{E}$, et al. Three-dimensional cell culture: a breakthrough in vivo. Int J Mol Sci 2015;16:1517-1527.

6. Clevers $\mathrm{H}$. Modeling development and disease with organoids. Cell 2016:165:1586-1597.

7. Buzhor E, Harari-Steinberg O, Omer D, et al. Kidney spheroids recapitulate tubular organoids leading to enhanced tubulogenic potency of human kidney-derived cells. Tissue Eng Part A 2011;17: 2305-2319.

8. Garcez PP, Loiola EC, Madeiro da Costa R, et al. Zika virus impairs growth in human neurospheres and brain organoids. Science 2016;352:816-818.

9. Sato $T$, Stange $D E$, Ferrante $M$, et al. Long-term expansion of epithelial organoids from human colon, adenoma, adenocarcinoma, and Barrett's epithelium. Gastroenterology 2011;141:1762-1772.

10. Ogawa $\mathrm{M}$, Ogawa $\mathrm{S}$, Bear $\mathrm{CE}$, et al. Directed differentiation of cholangiocytes from human pluripotent stem cells. Nat Biotechnol 2015;33:853-862.

11. Sampaziotis F, Cardoso de Brito M, Madrigal P, et al. Cholangiocytes derived from human induced pluripotent stem cells for disease modeling and drug validation. Nat Biotechnol 2015;8:843-855.

12. Tian $L$, Deshmukh $A$, Ye $Z$, et al. Efficient and controlled generation of $2 \mathrm{D}$ and $3 \mathrm{~B}$ bile duct tissue from human pluripotent stem cell-derived spheroids. Stem Cell Rev Rep 2016;12:500-508.

13. De Assuncao TM, Sun $Y$, Jalan-Sakrikar $N$, et al. Development and characterization of human-induced pluripotent stem cell-derived cholangiocytes. Lab Invest 2015;95:684-696.

14. Spirli C, Mariotti V, Villani A, et al. Adenylyl cyclase 5 links changes in calcium homeostasis to cAMP-dependent cyst growth in polycystic liver disease. J Hepatol 2016;66:571-580.

15. Waisbourd-Zinman $\mathrm{O}$, Koh H, Tsai S, et al. The toxin biliatresone causes mouse extrahepatic cholangiocyte damage and fibrosis through decreased glutathione and SOX17. Hepatology 2016;64:880-893.

16. Masyuk TV, Radtke BN, Stroope AJ, et al. Pasireotide is more effective than octreotide in reducing hepatorenal cystogenesis in rodents with polycystic kidney and liver diseases. Hepatology 2013;58:409-421.

17. Joplin R, Strain AJ, Neuberger JM. Immuno-isolation and culture of biliary epithelial cells from normal human liver. In Vitro Cell Dev Biol 1989;25:1189-1192.

18. Tabibian JH, Trussoni CE, O'Hara SP, et al. Characterization of cultured cholangiocytes isolated from livers of patients with primary sclerosing cholangitis. Lab Invest 2014;94:1126-1133.

19. Grubman SA, Perrone RD, Lee DW, et al. Regulation of intracellular $\mathrm{pH}$ by immortalized human intrahepatic biliary epithelial cell lines. Am J Physiol Gastrointest Liver Physiol 1994;266:G1060-G1070.

20. Kuroda $Y$, Wakao $S$, Kitada $M$, et al. Isolation, culture and evaluation of multilineage-differentiating stress-enduring (Muse) cells. Nat Protoc 2013;8:1391-1415.

21. Debnath J, Muthuswamy SK, Brugge JS. Morphogenesis and oncogenesis of MCF-10A mammary epithelial acini grown in threedimensional basement membrane cultures. Methods 2003;30:256-268.

22. Masyuk $\mathrm{Al}$, Huang $\mathrm{BQ}$, Ward $\mathrm{CJ}$, et al. Biliary exosomes influence cholangiocyte regulatory mechanisms and proliferation through interaction with primary cilia. Am J Physiol Gastrointest Liver Physiol 2010;299:G990-G999.

23. Mills KR, Reginato M, Debnath J, et al. Tumor necrosis factor-related apoptosis-inducing ligand (TRAIL) is required for induction of autophagy during lumen formation in vitro. Proc Natl Acad Sci USA 2004;101:3438-3443. 
24. Yu L, Leonardo MJ, Baehrecke EH. Autophagy and caspases: a new cell death program. Cell Cycle 2004;3:1124-1126.

25. Jung $\mathrm{SH}, \mathrm{H}$ wang $\mathrm{JH}$, Kim $\mathrm{SE}$, et al. Human galectin-9 on the porcine cells affects the cytotoxic activity of M1-differentiated THP-1 cells through inducing a shift in M2-differentiated THP-1 cells. Xenotransplantation 2017;24.

26. Dowal L, Parameswaran P, Phat S, et al. Intrinsic properties of brown and white adipocytes have differential effects on macrophage inflammatory responses. Mediators Inflamm 2017;2017:9067049.

27. Dianat N, Dubois-Pot-Schneider $\mathrm{H}$, Steichen $\mathrm{C}$, et al. Generation of functional cholangiocyte-like cells from human pluripotent stem cells and HepaRG cells. Hepatology 2014;60:700-714.
28. Tanimizu N, Miyajima A, Mostov KE. Liver progenitor cells develop cholangiocyte-type epithelial polarity in three-dimensional culture. Mol Biol Cell 2007;18:1472-1479.

29. Kido T, Koui Y, Suzuki K, et al. CPM is a useful cell surface marker to isolate expandable bi-potential liver progenitor cells derived from human iPS cells. Stem Cell Reports 2015;5: 508-515.

30. De Assuncao TM, Jalan-Sakrikar N, Huebert RC. Regenerative medicine and the biliary tree. Semin Liver Dis 2017;37:17-27.

31. Anzai K, Chikada H, Tsuruya $\mathrm{K}$, et al. Foetal hepatic progenitor cells assume a cholangiocytic cell phenotype during two-dimensional pre-culture. Sci Rep 2016;6:28283. 